\title{
Prolotherapy for the patients with chronic musculoskeletal pain: systematic review and meta-analysis
}

\section{Geonhyeong Bae, Suyeon Kim, Sangseok Lee, Woo Yong Lee, and Yunhee Lim}

Received September 23, 2020

Revised October 14, 2020

Accepted October 16, 2020

Department of Anesthesiology and Pain Medicine, Sanggye Paik Hospital, Inje University College of Medicine, Seoul, Korea

Background: Prolotherapy, which stimulates the healing of loosened ligaments and tendons, is a cost-effective and safe treatment modality for chronic musculoskeletal pain. Its benefits may be affected by injection protocols, comparative regimens, and evaluation scales. The aim of this study was to determine the effectiveness of dextrose prolotherapy as a long-term treatment for chronic musculoskeletal pain.

Methods: Medline, Embase, Cochrane Central, KoreaMed, and KMbase databases were searched for studies published up to March 2019. We included randomized controlled trials which compared the effect of dextrose prolotherapy with that of other therapies such as exercise, saline, platelet-rich plasma, and steroid injection. The primary outcome was pain score change during daily life.

Results: Ten studies involving 750 participants were included in the final analysis. Pain scores from 6 months to 1 year after dextrose prolotherapy were significantly reduced compared to saline injection (standardized mean difference [SMD] -0.44; 95\% confidence interval $[\mathrm{Cl}]-0.76$ to $-0.11, \mathrm{P}=0.008)$ and exercise (SMD $-0.42 ; 95 \% \mathrm{Cl}-0.77$ to $-0.07, \mathrm{P}=$

\section{Corresponding author}

Yunhee Lim, M.D., Ph.D.

Department of Anesthesiology and Pain Medicine, Sanggye Paik Hospital, Inje University College of Medicine, 1342 Dongil-ro, Nowon-gu, Seoul 01757 , Korea

Tel: 82-2-350-1176

Fax: 82-2-950-1323

E-mail: painfree@paik.ac.kr
0.02). Prolotherapy yielded results similar to platelet-rich plasma or steroid injection, that it showed no significant difference in pain score.

Conclusions: Dextrose prolotherapy is more effective in the treatment of chronic pain compared to saline injection or exercise. Its effect was comparable to that of platelet-rich plasma or steroid injection. Adequately powered, homogeneous, and longer-term trials are needed to better elucidate the efficacy of prolotherapy.

Keywords: Musculoskeletal pain; Platelet-rich plasma; Prolotherapy; Steroids.

\section{INTRODUCTION}

Chronic musculoskeletal pain is defined as pain that lasts for three to six months or beyond the time of normal healing [1]. Musculoskeletal disorders are the most common source of chronic musculoskeletal pain, and their in- creasing prevalence has led to a need for effective non-surgical solutions, such as physical therapy, pharmacologic treatment, and injection-based treatment [2]. Injection therapies can be introduced when pain or functional limitations are significant despite oral medication or exercise [3]. Corticosteroid injections are the most common regi-

This is an Open Access article distributed under the terms of the Creative Commons Attribution Non-Commercial License (http://creativecommons.org/licenses/by-nc/4.0) which permits unrestricted non-commercial use, distribution, and reproduction in any medium, provided the original work is properly cited.

Copyright (c) the Korean Society of Anesthesiologists, 2021 
men for musculoskeletal disorders; they provide shortterm symptomatic improvement, but aggravate cartilage damage, thus increasing the risk of tissue atrophy [4]. Therefore, physicians have become interested in alternative injectants, such as prolotherapy or platelet-rich plasma (PRP) [5].

Prolotherapy is a nonsurgical regenerative injection technique that administers small amounts of an irritant solution to the degenerated tendon insertions (entheses), joints, ligaments, and adjacent joint spaces over a series of several treatment sessions [6-8]. The mechanism of action behind prolotherapy is not completely understood, but the current theory is that the injected proliferate causes a healing process that is similar to the body's natural healing process, whereby a local inflammatory cascade is initiated, which triggers the release of growth factors and collagen deposition [2]. To date, many studies which support the benefits of the use of prolotherapy in patients with chronic musculoskeletal pain have been reported $[9,10]$. However, few meta-analyses have analyzed the effect of prolotherapy in patients with chronic musculoskeletal pain. Therefore, we designed a meta-analysis to evaluate the effect of prolotherapy in the treatment of chronic musculoskeletal pain and compare the effect of prolotherapy with other treatments.

\section{MATERIALS AND METHODS}

\section{Study design}

This meta-analysis was performed according to the recommendations of the PRISMA and Cochrane Collaboration. The protocol was registered with PROSPERO (no. CRD42019130609).

\section{Information sources and search strategy}

Two reviewers (WL, YL) systematically searched electronic databases such as Medline, Embase, and the Cochrane Library (CENTRAL) with no limitations on the year of publication. Additionally, KoreaMed (https://koreamed. org) and KMbase (http://kmbase.medric.or.kr) were used to search for manually relevant domestic articles. Broad search terms such as "prolotherapy", "chronic osteoarthritis", and "randomized controlled trials", were included to achieve higher sensitivity, and Medical Subject Heading (MeSH) terms were used. The languages of the articles were limited to Korean and English. The last search was conducted on March 10, 2019.

We did not search grey literature, despite its important contribution to a systematic review, because we wanted to present an effective basis for treatment to clinicians with as little bias as possible, based on the results of RCTs.

\section{Study selection and eligibility criteria}

All relevant studies were independently screened by two reviewers (WL and YL). Selection of relevant articles was done primarily at the title and abstract level, then after at the full-text level. Studies for the final assessment were selected based on the agreement of the two reviewers. Any disagreement was resolved by discussion with a third reviewer (SL).

Studies were included in the meta-analysis if they satisfied the following criteria: (1) patients with chronic musculoskeletal pain lasting for more than 3 months; (2) prolotherapy using dextrose for any joints, tendon, and/or ligaments; (3) results of the non-prolotherapy group were reported; and (4) the post-injection pain score was reported as the primary outcome.

Studies were excluded for the following reasons: (1) use of prolotherapy solutions containing anything other than glucose (polidocanol, manganese, zinc, human growth hormone, phenol-glucose-glycerine, pumice, ozone, glycerin, phenol, PRP, bone marrow, lipoaspirate, stem cells, or sodium morrhuate); (2) injection into the epidural space; (3) did not report appropriate outcomes or outcome measurements as mentioned; (4) non-randomized controlled trials; (5) non-human studies; (6) articles not in English or Korean.

\section{Risk of bias in individual studies}

Two independent authors (WL and $\mathrm{YL}$ ) reviewed the articles to assess the risk of bias (ROB) using the ROB tool provided in the Review Manager software version 5.3 (The Cochrane Collaboration, UK) based on Cochrane's assessment of the risk of bias [11]. If necessary, a third reviewer (SL) was included in the discussion to sort out the disagreements. The following eight domains were used to assess the risk of bias in each trial: random sequence generation (selection bias), allocation concealment (selection bias), blinding of participants and personnel (performance bias), blinding of outcome assessment (detection bias), in- 
complete outcome data (attrition bias), selective reporting (reporting bias), and other bias. The methodology for each trial was graded as "high", "low", or "unclear" to reflect a high risk of bias, low risk of bias, or uncertainty of bias, respectively. The agreement between the two independent reviewers for the level of risk of bias regarding the eight domains was assessed using Cohen's kappa. Kappa values were interpreted as follows: 1 ) less than 0: less than chance agreement, 2) 0.01 to 0.20 : slight agreement, 3) 0.21 to 0.40 : fair agreement, 4) 0.41 to 0.60 : moderate agreement, 5) 0.61 to 0.80 : substantial agreement, and 6) 0.8 to 0.99 : almost perfect agreement.

\section{Data collection process and extracted items}

Two authors (WL and YL) extracted data from the original articles, and another author (SL) independently confirmed all of the extracted data. The general characteristics (i.e., the study design, publication year, and name of the first author), intervention types and methods, and outcomes were extracted for each study based on the inclusion criteria. Each method of the intervention, such as the prolotherapy regimen, interval, and duration, was extracted. The measured outcomes included the number of patients analyzed in each group, tools for pain assessment, and pain scores.

The main outcome was determined by the severity of the pain, derived from the results of the pain scale. The first priority of pain measurement extraction was the pain score for 6 months to 1 year. To assess the effectiveness of dextrose prolotherapy, we used the standardized mean difference of pain scores between the prolotherapy group and other comparator groups using exercise, saline, PRP, and steroid injection.

\section{Subgroup analysis}

We grouped the analyses of VAS for pain into less than three months, three to six months, and more than six months while registering our review in PROSPERO. However, we were unable to classify the subgroups as originally planned because not all the individual studies followed the patients and reported the resulting variables on these criteria. Using the common denominator of the results of the individual studies, we were able to synthesize results that could be divided into three subgroups: baseline to 1 month, 1 month to 3 months, and 6 months to 1 year.

\section{Statistical analysis}

Continuous data (e.g., post-injection pain scores) were pooled as standardized mean differences (SMDs) because different outcome measurement scaling was expected across trials. We also calculated the $95 \%$ confidence intervals (CIs) for all estimates. A random-effect model was used to pool the study results, taking into account possible variations in effect sizes across trials. The heterogeneity statistic Cochrane Q and its corresponding degrees of freedom (df) and P value, as well as Higgins' I2 as a measure of heterogeneity were calculated. $\mathrm{P}$ values $<0.05$ were considered to be representative of statistically significant heterogeneity, and I 2 values $>50 \%$ were considered to represent significant heterogeneity. Post-hoc subgroup analyses were performed where possible for each outcome to explore heterogeneity based on the different sites of injection. Chi-squared tests for heterogeneity were performed to identify differences between subgroups. Publication bias was not evaluated because only a few $(<10)$ studies were included in this meta-analysis. We conducted a sensitivity analysis to evaluate the influence of each study on the long-term (six months to one year) therapeutic effect of prolotherapy compared with saline by excluding one trial at a time from the pooled effects. All analyses were performed using R 3.51 (R Foundation for Statistical Computing, Austria) and Review Manager (RevMan, version 5.3, The Cochrane Collaboration).

\section{RESULTS}

\section{Study selection and characteristics}

We retrieved 680 articles after the initial database search: Medline ( $\mathrm{n}=250)$, EMBASE $(\mathrm{n}=64)$, CENTRAL $(\mathrm{n}=168)$, and Korean databases $(\mathrm{n}=198)$.

After excluding 567 duplicate articles, primary selection was performed on 131 articles. First, we excluded 66 unrelated articles based on titles and abstracts. Second, we excluded 27 articles that only included abstracts. Thereafter, full-text reviews were conducted for 38 articles. Of these 38 full-text articles, 28 were excluded for the following reasons: not controlled with placebo or other treatment $(\mathrm{n}=$ 14), patients' pain period not clearly described or less than three months $(\mathrm{n}=9)$, duplication $(\mathrm{n}=4)$, and articles not in English or Korean $(n=1)$. The reasons for exclusion of these papers are given in detail in Table 1. Finally, ROB 

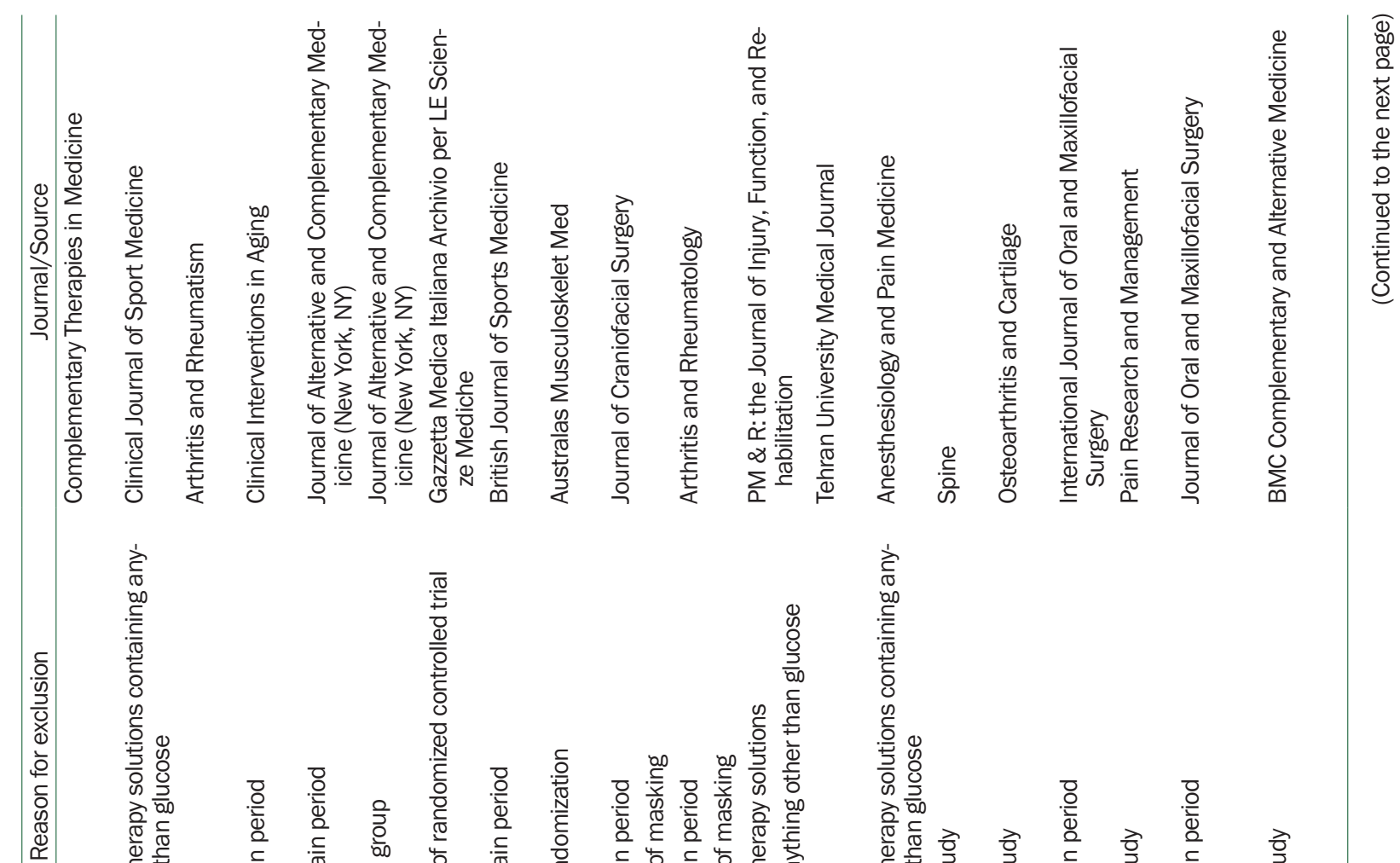

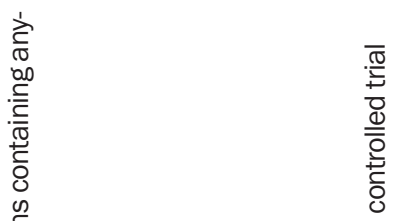
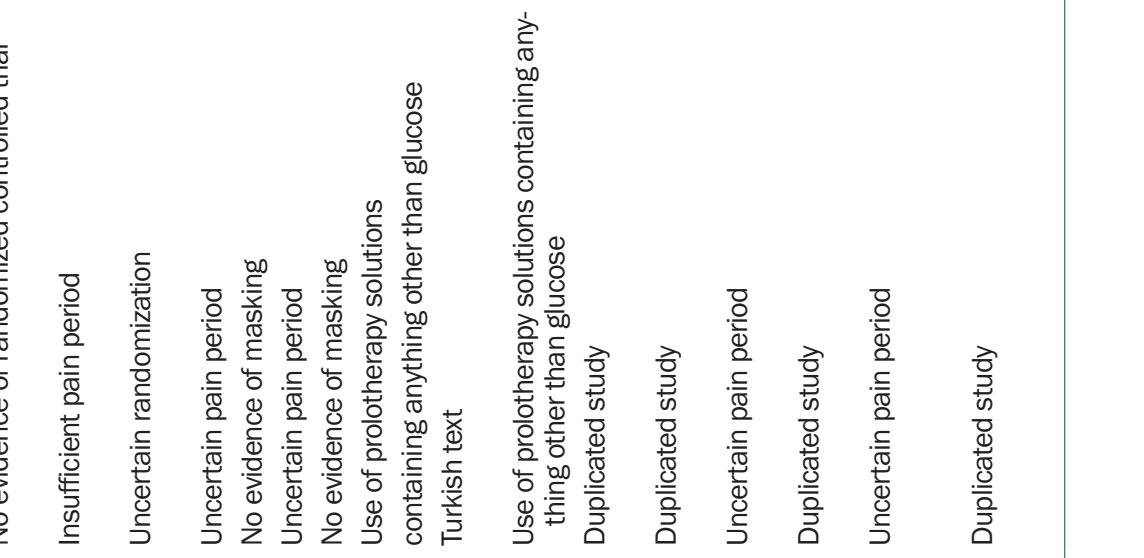

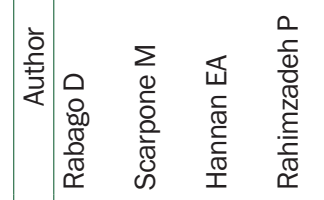

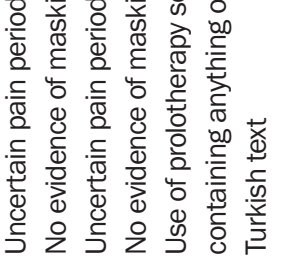

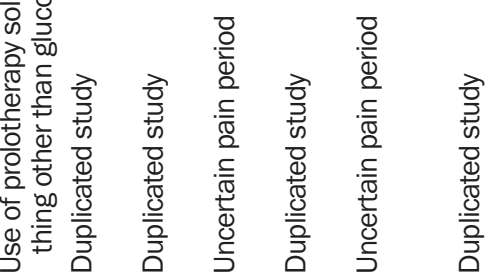

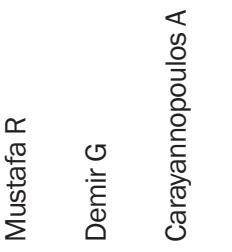

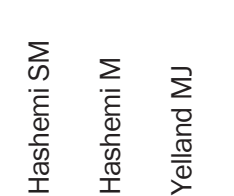

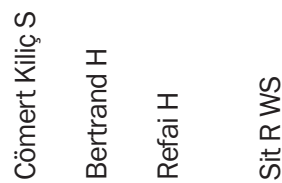

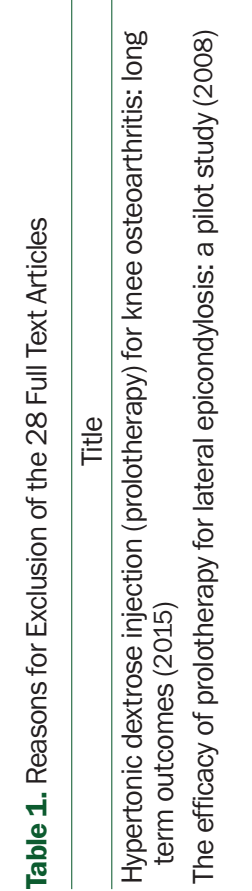

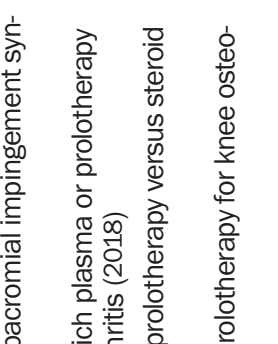

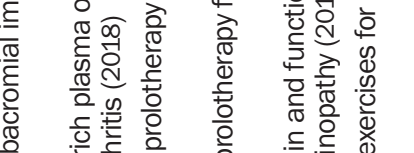

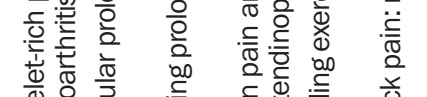

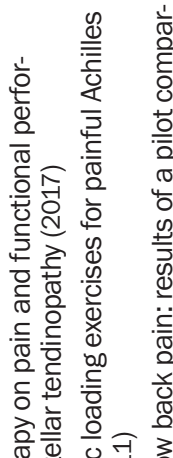

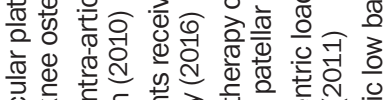

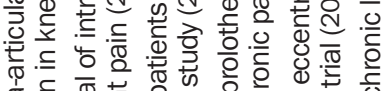

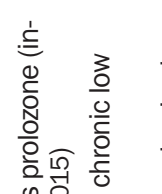

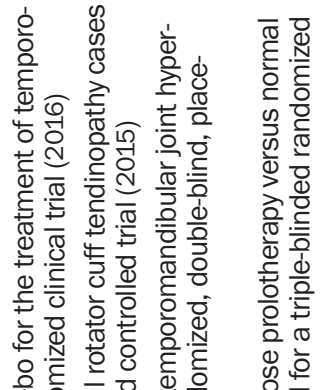

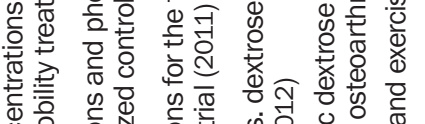

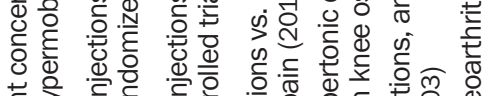

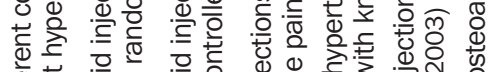

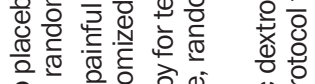

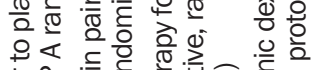

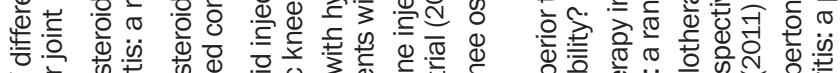

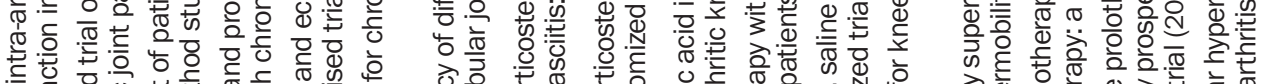

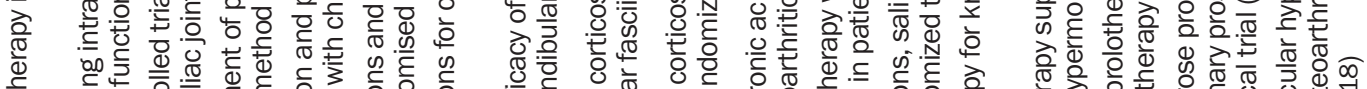

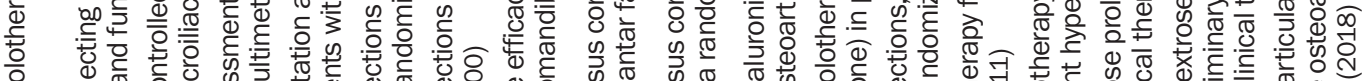

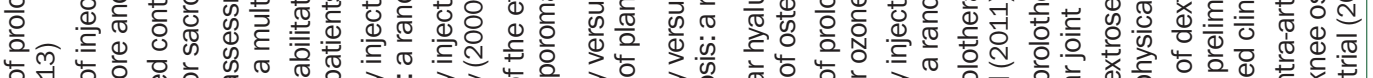

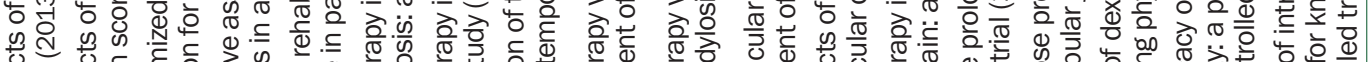

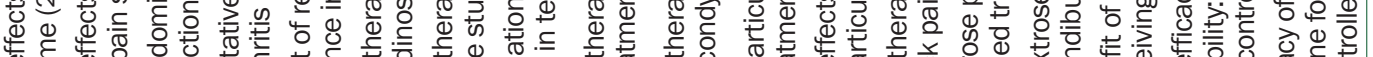

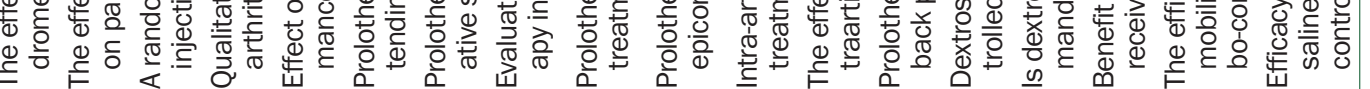




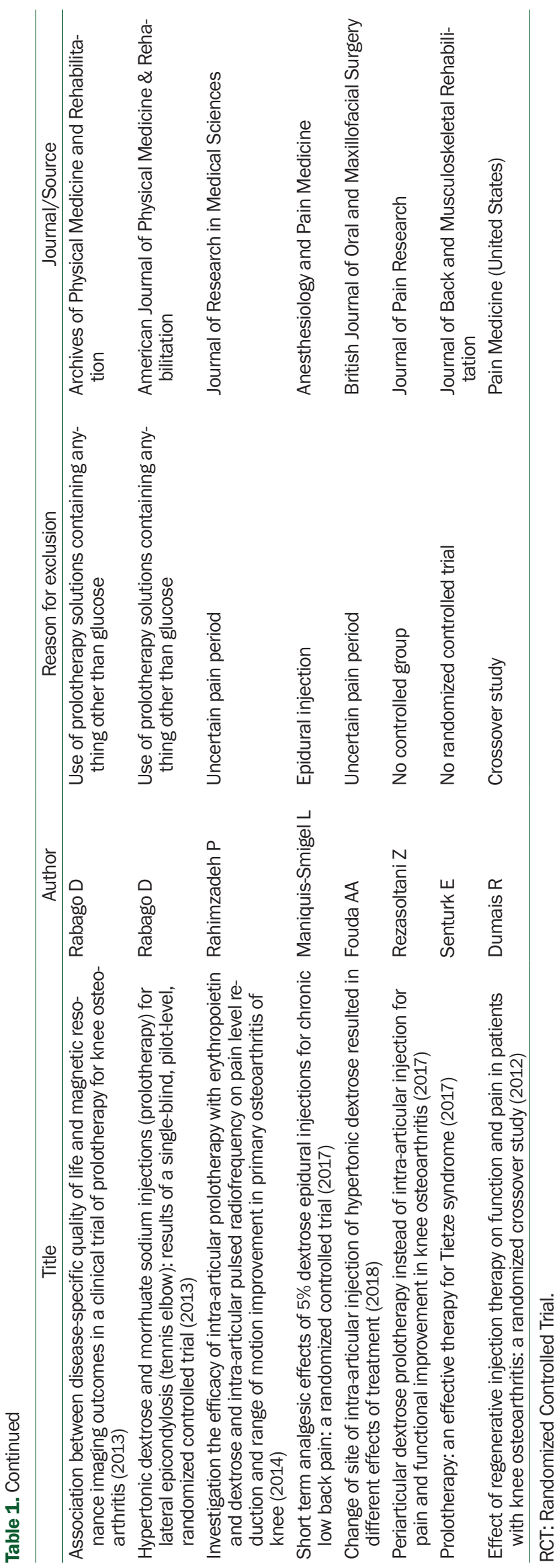

evaluation and data extraction were performed on 10 articles (Fig. 1).

All studies were randomized controlled trials. Various injection sites, including large joints such as the knee and small joints such as finger joints and carpometacarpal joints were investigated. The comparator groups were saline injection, exercise, steroid injection, PRP injection, and extracorporeal shock wave therapy. The severity of pain as the primary outcome was measured using the Visual Analog Scale (VAS), the Western Ontario and McMaster Universities Osteoarthritis Index, the Karnofsky Performance Score, and Foot Function Index. The concentration, volume of dextrose solution, and interval between injection sessions were different between studies. The dextrose concentration ranged from $5 \%$ to $25 \%$, and the injection interval ranged from weeks to months (Table 2).

\section{Quality assessment of the included studies (risk of bias within studies)}

ROB evaluation revealed an overall low risk for selection and reporting bias, while almost half of the studies showed a high risk of performance bias because they could not be blinded to the differences in procedures (Figs. 2, 3). All studies reported detailed information regarding the randomization techniques that were used, such as manual random number selection or a computer-generated random number table. Allocation concealment was unclear in five studies which did not mention the specific allocation concealment method.

For performance bias, four studies [12-15] were unable to blind the participants and five studies [12-14,16,17] could not blind the physicians because the injection site was different or because exercise was included in a control group. These studies were considered "high" bias. Two studies had high detection bias $[15,17]$.

The risk of incomplete outcome data was "high" in four studies that did not mention a minimal sample size $[15,16,18,19]$. Four other studies did not meet a minimal sample size, resulting in an unclear risk of bias $[14,17,20,21]$.

Reporting bias was low because there was no selective reporting in any of the studies. Regarding the potential bias, four studies were rated as unclear because there was no detailed description of the sample size calculation, and one study was high risk because the number of samples was very small [17]. 


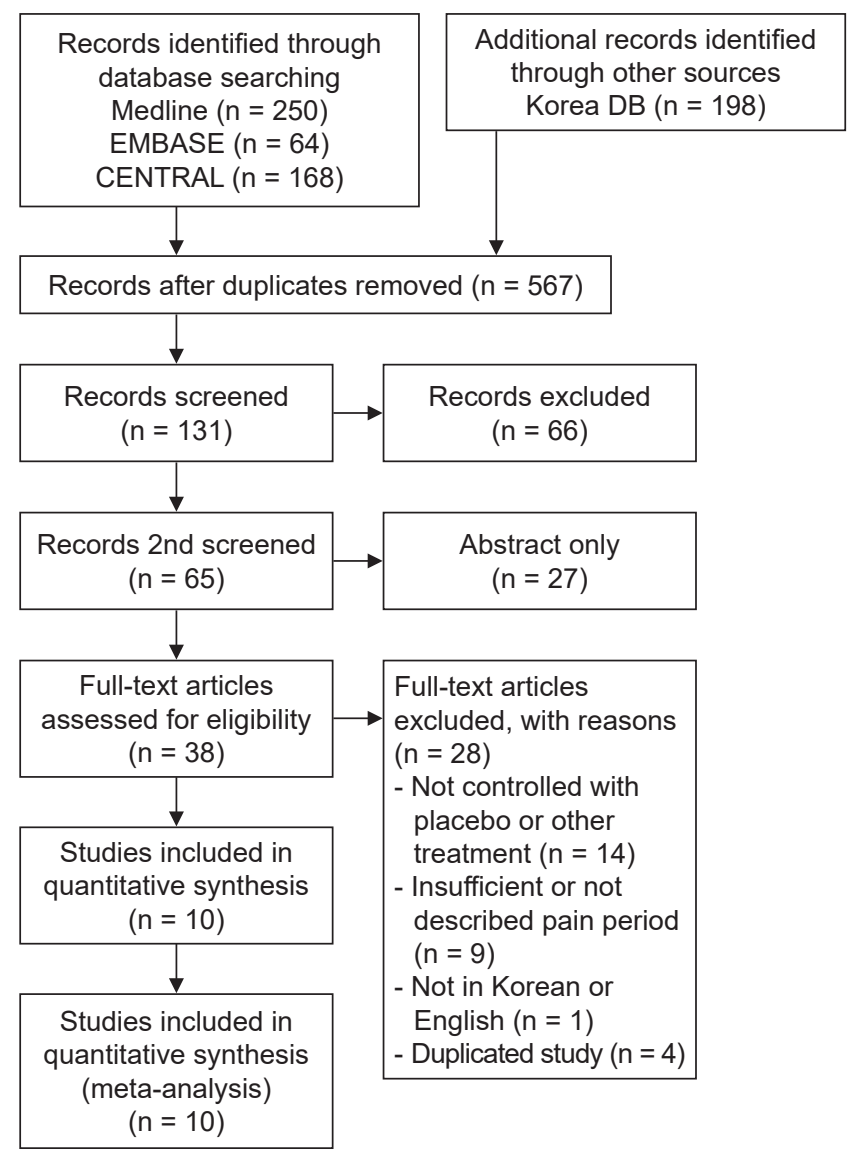

Fig. 1. PRISMA flow diagram. Flow diagram of search strategy and study selection. DB: database.

The kappa value between the two reviewers for the 10 selected articles was 0.81 .

\section{Effectiveness of prolotherapy compared with other therapies}

\section{Prolotherapy with dextrose compared to saline}

The effectiveness of prolotherapy compared to saline was reported in five studies $[15,16,18-20]$ ( $\mathrm{n}=246$; prolotherapy group $=126$, normal saline group $=120$ ), which suggested that prolotherapy with dextrose significantly reduced the pain score from 6 months to 1 year (SMD, -0.44 ; $95 \%$ CI $[-0.76$ to -0.11$]$; $\mathrm{P}=0.008$; $\mathrm{I}^{2}=36 \%$; Fig. $4 \mathrm{~A}$ ). However, there was no difference between the effects of both therapies during the other periods analyzed (SMD, 0.42; 95\% CI [0.51 to 1.35]; $\mathrm{P}=0.003 ; \mathrm{I}^{2}=88 \%$ at baseline to 1 month; SMD, -0.07 ; 95\% CI [ -0.37 to 0.23$]$; $\mathrm{P}=0.66$; $\mathrm{I}^{2}=$ $0 \%$ at 1 month to 3 months). Sensitivity analysis using a single study removal method did not significantly change the pooled results. The therapeutic effect of prolotherapy was $33 \%$ lower (SMD, -0.29 ; $95 \%$ CI $[-0.57$ to -0.01$]$; $\mathrm{P}=$ 0.040 ) than the pooled estimate effect size (SMD, -0.44 ; 95\% CI [ -0.91 to -0.13$] ; \mathrm{P}=0.009$ ) after omitting one trial [16].

\section{Prolotherapy with dextrose compared to exercise}

Two studies $[15,18](n=128$; prolotherapy group $=63$, exercise group $=65)$ provided data on pain scores comparing prolotherapy and exercise. Compared to exercise, dextrose therapy significantly reduced the pain score from 1 month to 3 months (SMD, $-0.44 ; 95 \%$ CI [ -0.84 to -0.04 ]; P $\left.=0.11 ; \mathrm{I}^{2}=55 \%\right)$ and 6 months to 1 year (SMD, $-0.42 ; 95 \%$ CI [ -0.77 to -0.07$] ; \mathrm{P}=0.02 ; \mathrm{I}^{2}=0 \%$; Fig. $4 \mathrm{~B}$ ). However, there was no difference in the effects of both therapies during the baseline to 1-month-period (SMD, -0.42; 95\% CI [-1.14 to 0.30]; $\mathrm{P}=0.02 ; \mathrm{I}^{2}=83 \%$ ).

\section{Prolotherapy with dextrose compared to PRP}

Two studies [12,17] $(\mathrm{n}=99$; prolotherapy group $=51$, PRP group $=48$ ) reported data on pain scores comparing prolotherapy and PRP. Prolotherapy with dextrose had a therapeutic effect corresponding to that of PRP, and there was no significant difference from 1 month to 3 months (SMD, $0.05 ; 95 \% \mathrm{CI}[-0.34$ to 0.45$] ; \mathrm{P}=0.96 ; \mathrm{I}^{2}=0 \%$ ) and 6 months to 1 year (SMD 0.19 ; $95 \%$ CI [ -0.20 to 0.59 ]; $\mathrm{P}=$ 0.34 ; $\mathrm{I}^{2}=0 \%$; Fig. 4C).

\section{Prolotherapy with dextrose compared to a steroid}

Two studies $[12,21](\mathrm{n}=135$; prolotherapy group $=$ 68 , steroid group $=67$ ) suggested that prolotherapy with dextrose had a therapeutic effect comparable to that of steroids from 1 month to 3 months (SMD, 0.22; 95\% CI [ -1.27 to 1.70$\left.] ; \mathrm{P}<0.001 ; \mathrm{I}^{2}=94 \%\right)$ and 6 months to 1 year (SMD, $0.45 ; 95 \%$ CI [0.57 to 1.47 ]; $\mathrm{P}=0.39 ; \mathrm{I}^{2}=88 \%$; Fig. 4D).

\section{DISCUSSION}

Previous studies have reported that prolotherapy is effective for treating musculoskeletal pain. However, their analyses included a small number of studies, which was not thought to be enough to compare prolotherapy with common regimens such as corticosteroids or PRP [2,22].

Our principal findings revealed that prolotherapy with dextrose has a clear and positive effect on chronic musculoskeletal pain ranging from 6 months to 1 year. In comparison with saline injection or exercise, treatment with pro- 


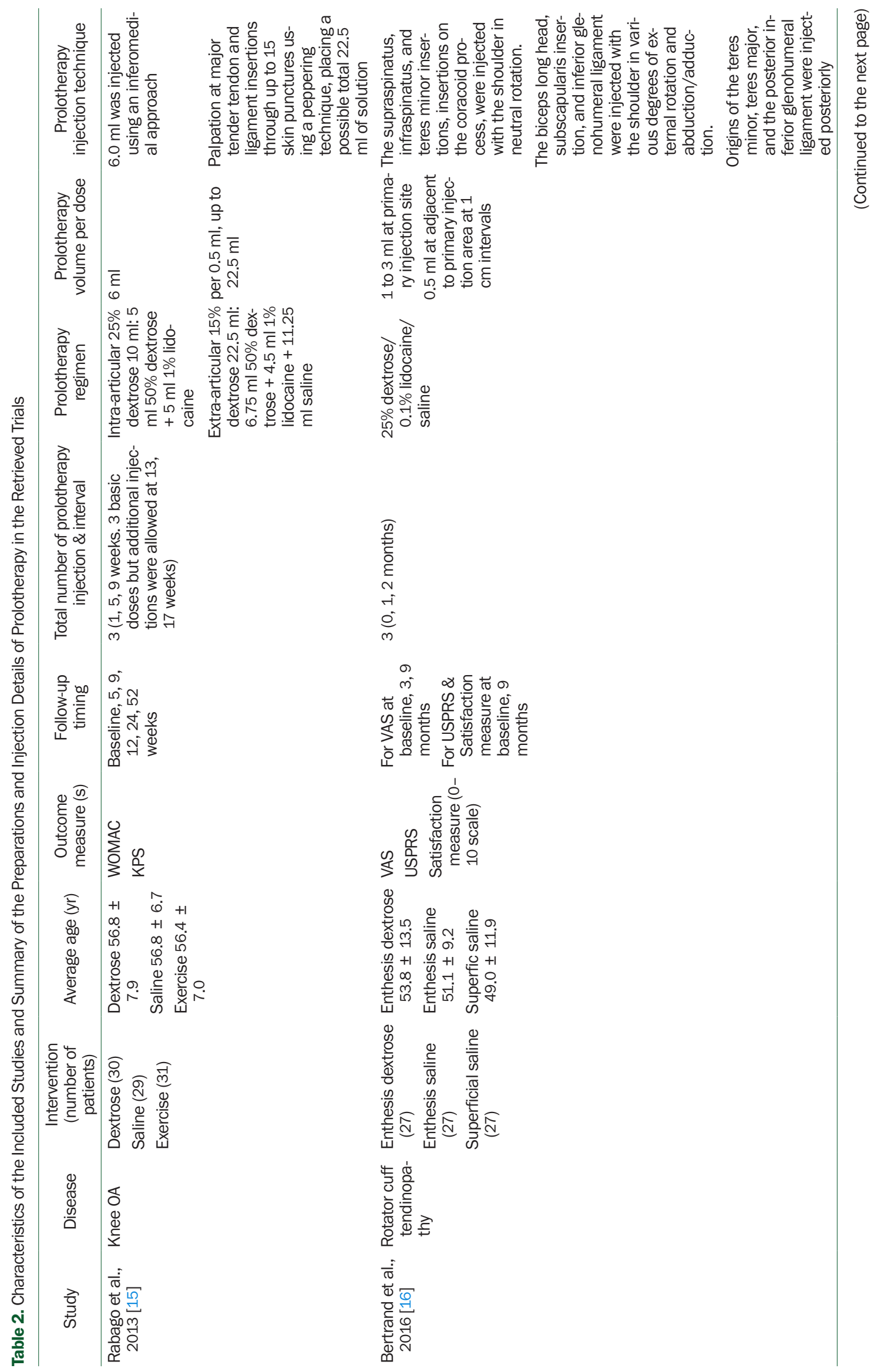




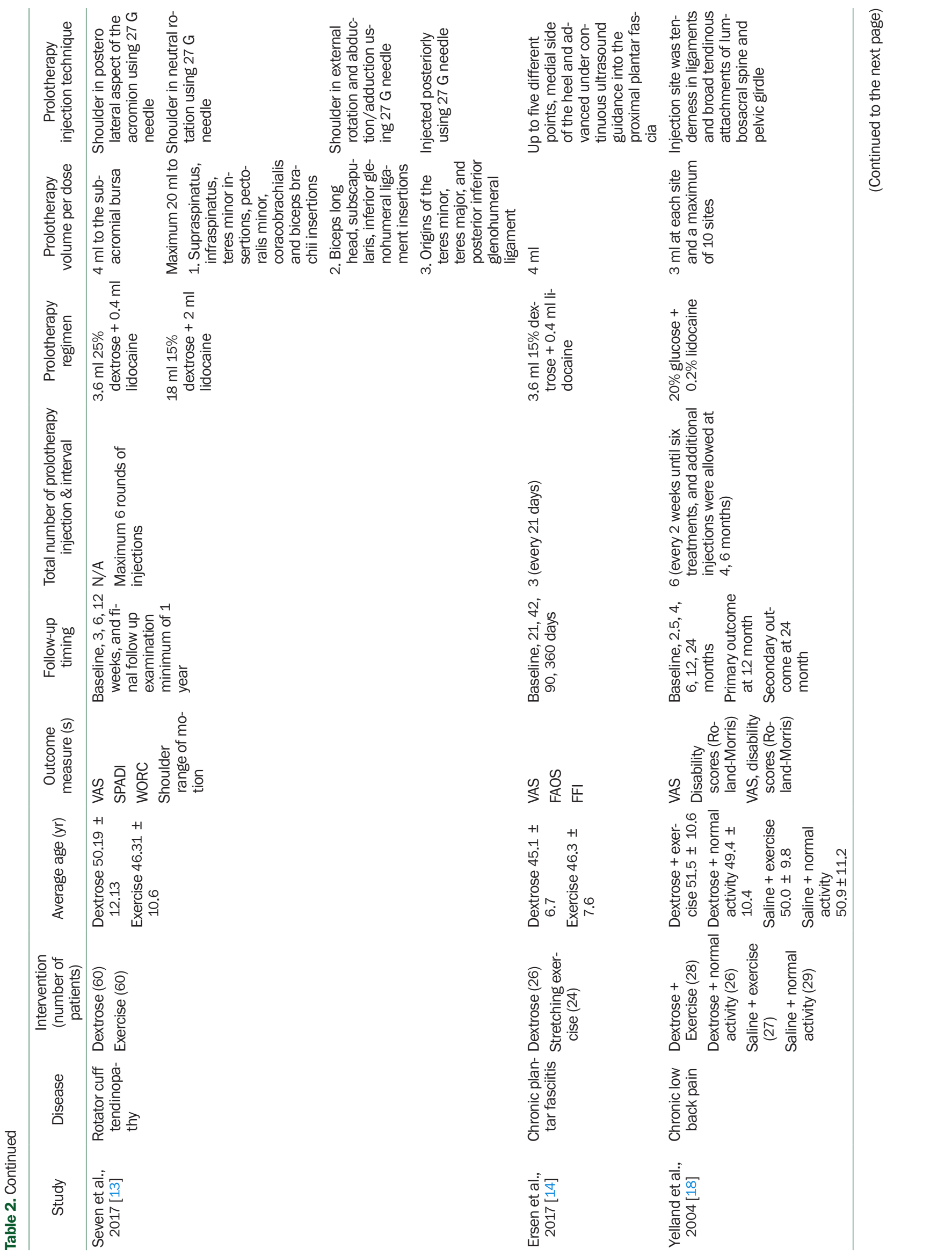




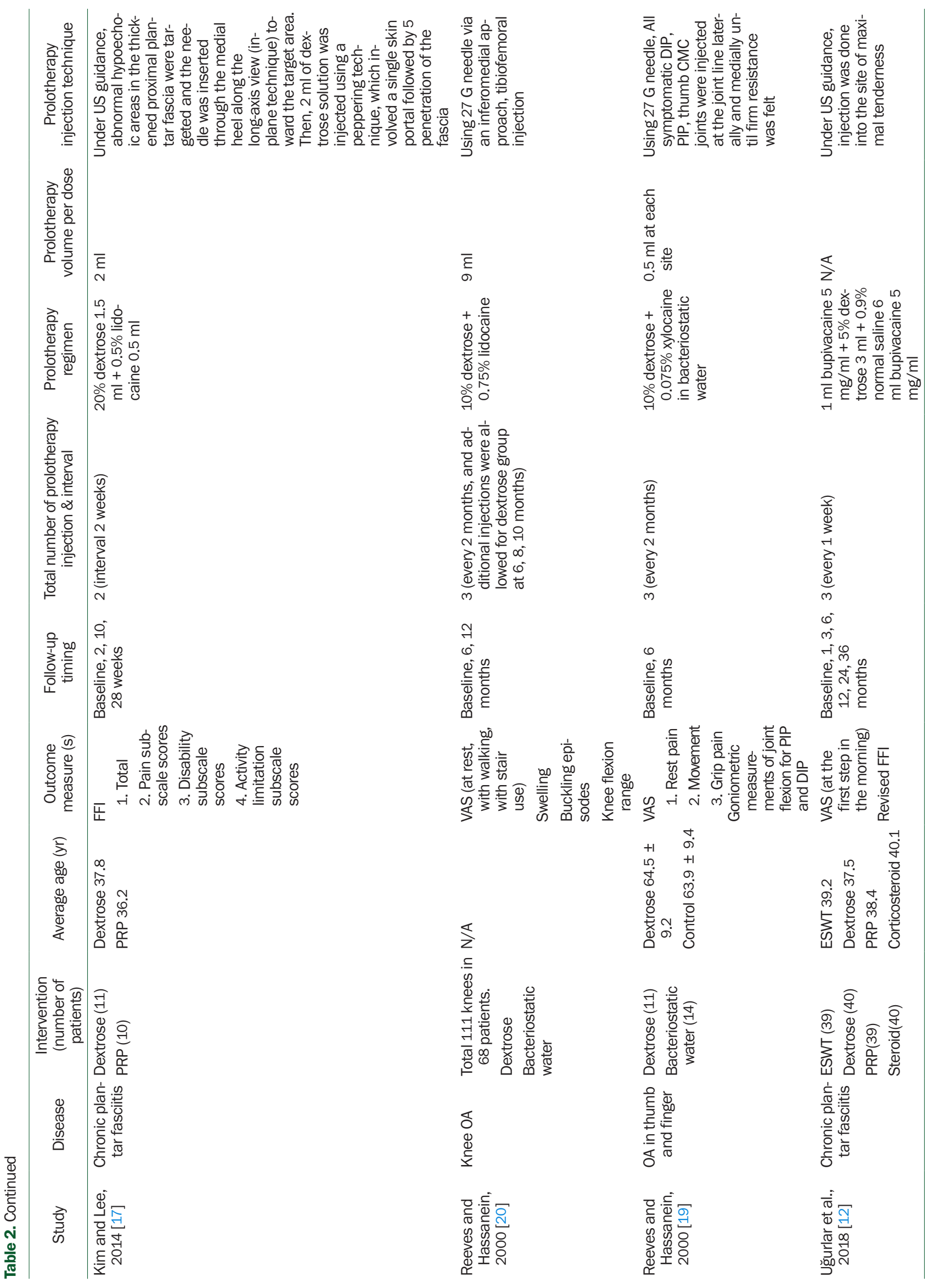




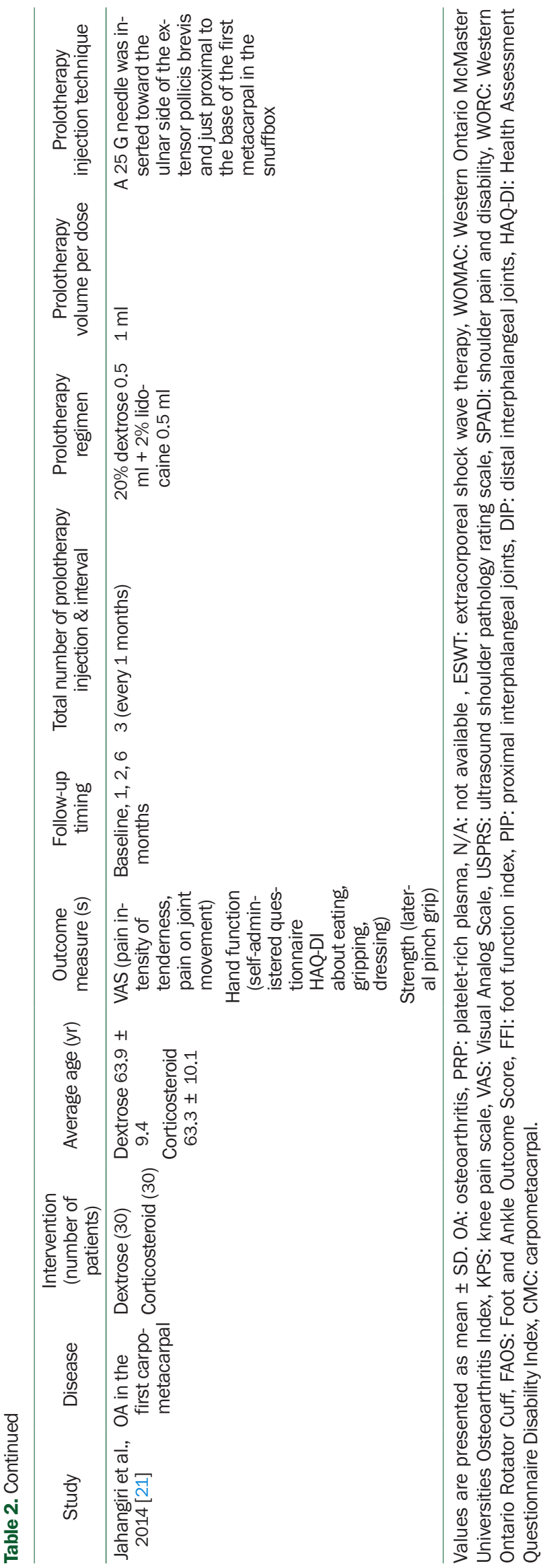

after treatment.

Corticosteroid injection has been widely used as it is known to be effective in the treatment of musculoskeletal disorders. In vitro studies have shown that corticosteroids have therapeutic effects on the tendon and the surrounding connective tissues by inhibiting collagen, extracellular matrix molecules, and granulation tissue production, in addition to inflammatory suppression [23]. However, such positive therapeutic effects of corticosteroids may exist only in the short term [24]. Uğurlar et al. [12] reported that corticosteroid injection was an effective treatment in the first 6 months, but lost its effectiveness after the first 6 months. The effect of pain relief in prolotherapy was seen within 3 to 12 months. In another study, Jahangiri et al. [21] compared the effects of corticosteroid injection and prolotherapy in patients with first carpometacarpal osteoarthritis and reported that the corticosteroid injection group had better results of pain score at 1 month. However, after 2 months, prolotherapy had a more favorable outcome than corticosteroid injection. Although not shown in our study, another concern of corticosteroids is adverse effects, such as focal inflammation, necrosis, fragmentation of collagen bundles in the subacromial space, tendon/ligament weakening or rupture, and worsening osteoarthritic changes [25-27]. In contrast, prolotherapy has no serious side effects and is effective, safe, and sustainable [10]. In this study, three RCTs reported only minor transient complications such as mild to moderate pain and self-limiting bruising after prolotherapy.

We found that PRP and dextrose prolotherapy were shown to be effective for treating degenerative conditions and injuries. Both PRP therapy and prolotherapy commonly have regenerative therapeutic properties, but the central mechanisms of prolotherapy and PRP are different. In prolotherapy, hyperosmolar dextrose triggers an inflammatory response, increases platelet-derived growth factor expression, and upregulates several mitogenic factors that may act as signaling mechanisms in tendon repair [28-30]. In PRP therapy, it aims to augment the natural healing process of tendon repair and regeneration by delivering high concentrations of growth factors directly to a lesion [31]. For preparation, following the extraction of autologous venous blood with a large-gauge needle to prevent premature platelet activation [32], platelets are separated from other blood components and further concentrated [33]. This occurs through a centrifuge process, in which platelets can be isolated from the other cell components of blood based on 


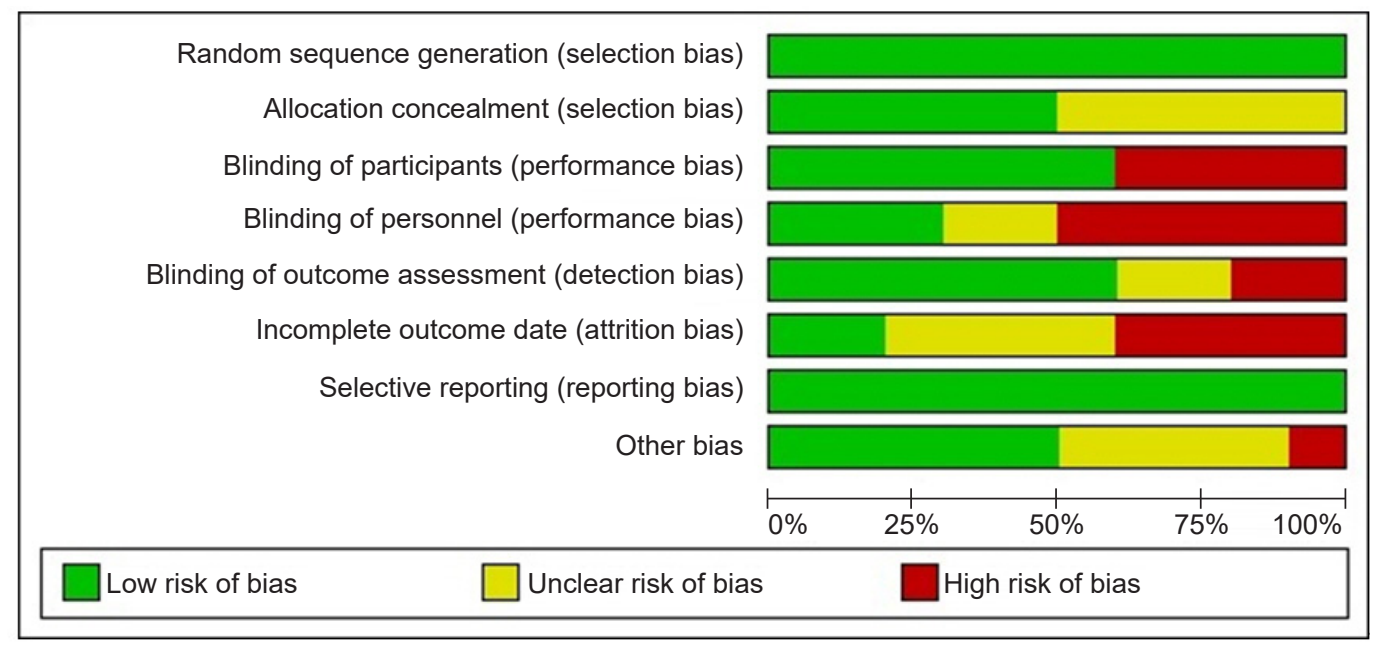

Fig. 2. Risk of bias graph. Risk of bias summary: review authors' judgements about each risk of bias item for each included study.

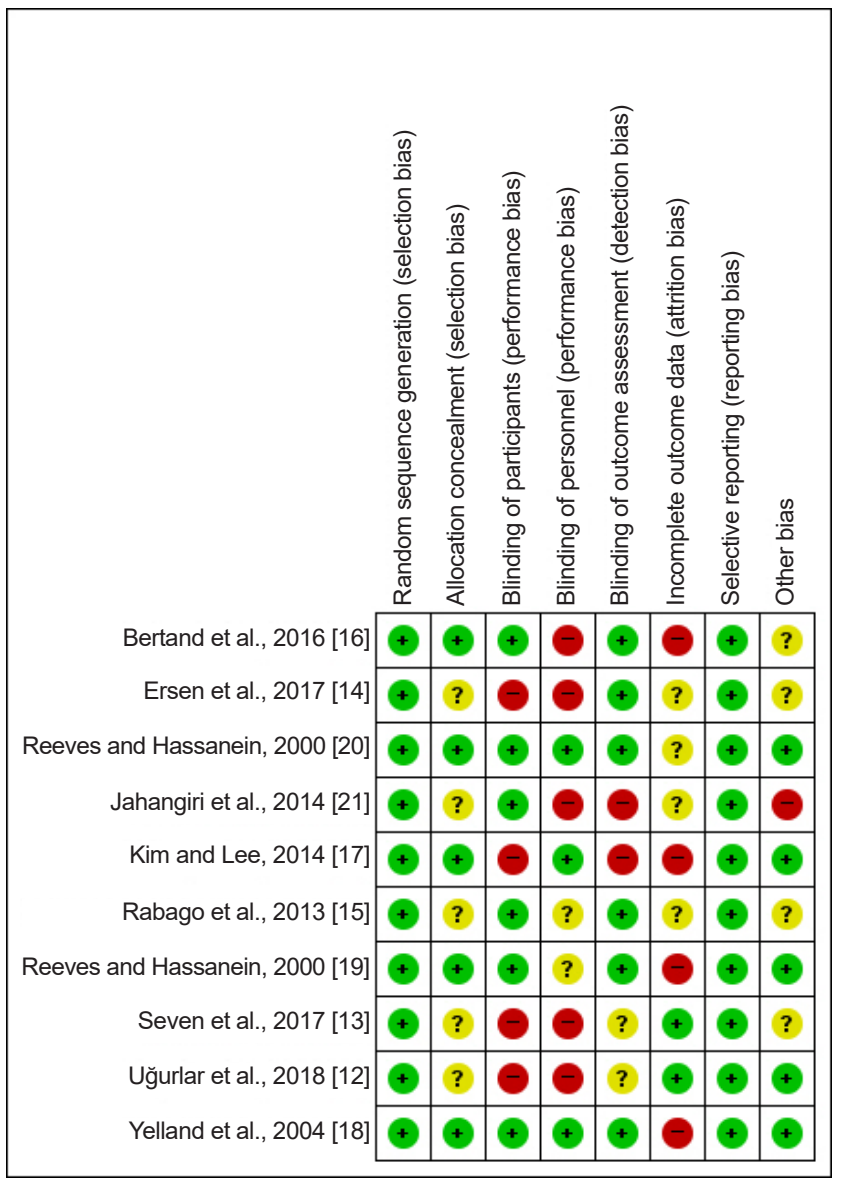

Fig. 3. Risk of bias summary. Risk of bias graph: review authors' judgements about each risk of bias item presented as percentages across all included studies.

lotherapy showed a moderately superior therapeutic effect. In particular, prolotherapy was found to be more effective than exercise from one month after treatment. It was also found to have a similar effect to steroids or PRP one month their physiological size [33]. Further concentration of platelets occurs with subsequent centrifuge cycles [34]. As such, several steps are needed to prepare PRP, whereas the preparation of the prolotherapy is simple. And PRP involves an invasive procedure (i.e., blood drawing) and lacks an optimized standardized protocol. In this regard, prolotherapy can provide more convenience to both patients and treatment providers.

Of the ten papers included in the study, nine papers showed generally positive results of achieving pain relief and patient satisfaction regardless of the injection site. Yelland et al. [18] reported that prolotherapy was not more effective than injections of normal saline for low back pain. Nevertheless, participants exhibited marked and sustained improvements in their pain and disability, even with saline injections. They assumed that these therapeutic effects could be achieved by other factors such as patients were enrolled in a trial during severe pain and then spontaneously recovered naturally, or by the therapeutic effect by direct needling of entheses, or the placebo effect by clinical visits.

In the case of using physiotherapy as a control group $[13,14]$, the positive result from the comparison with prolotherapy was within expectations because injection carries a strong placebo effect, which usually leads to a superior response to the noninvasive treatment.

The present study mainly analyzed the pain measurement outcomes, and functional improvement measurements were not considered. Among the RCTs, investigations of functional improvements were conducted in eight studies. Six studies reported that the prolotherapy group 
A. Dextose vs. Saline on VAS for Pain Composite 6 months-1 year (SMD)

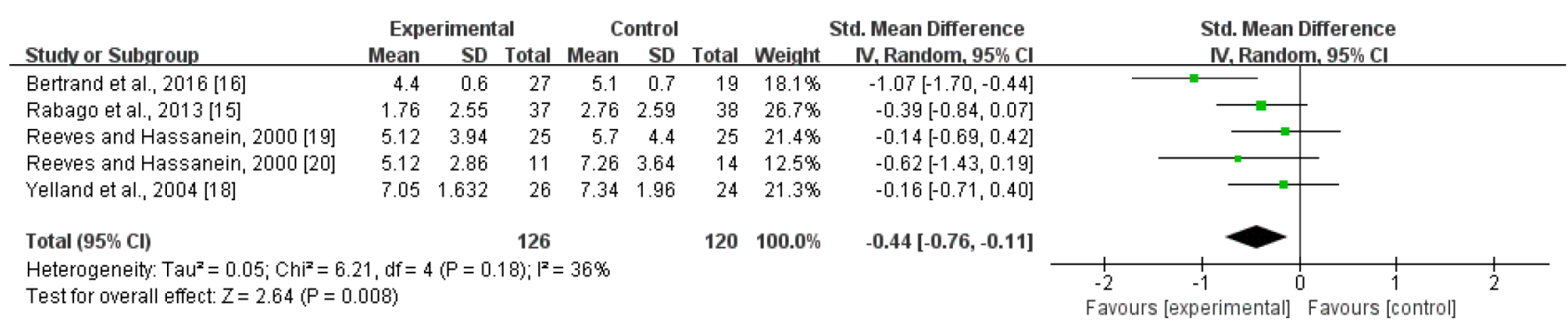

\section{B. Dextose vs. Exercise on VAS for Pain Composite 6 months-1 year (SMD)}

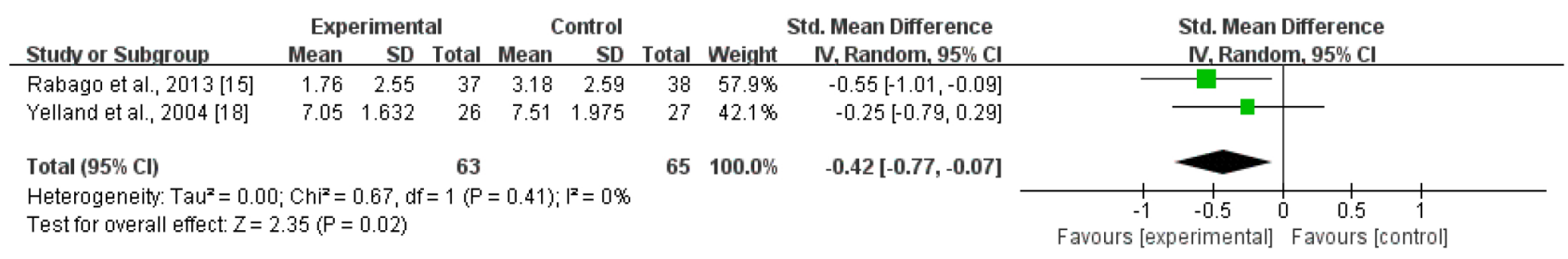

C. Dextose vs. Platelet-rich plasma on VAS for Pain Composite 6 months-1 year (SMD)

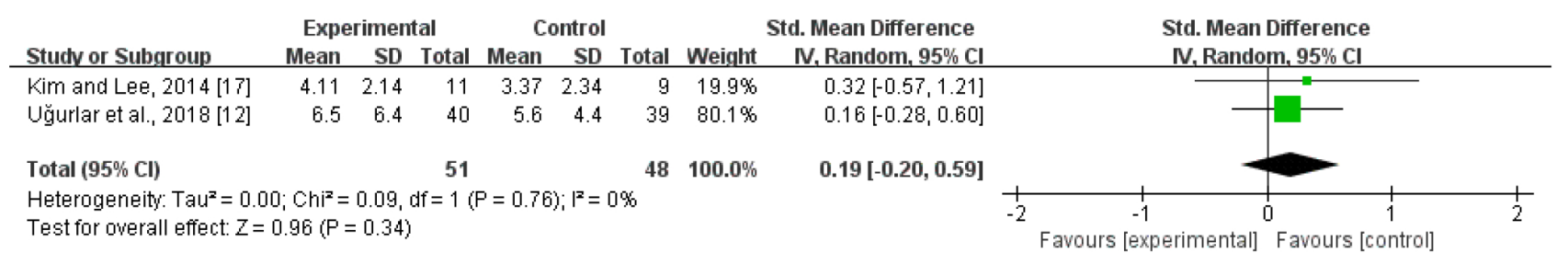

\section{Dextose vs. Steroid on VAS for Pain Composite 6 months-1 year (SMD)}

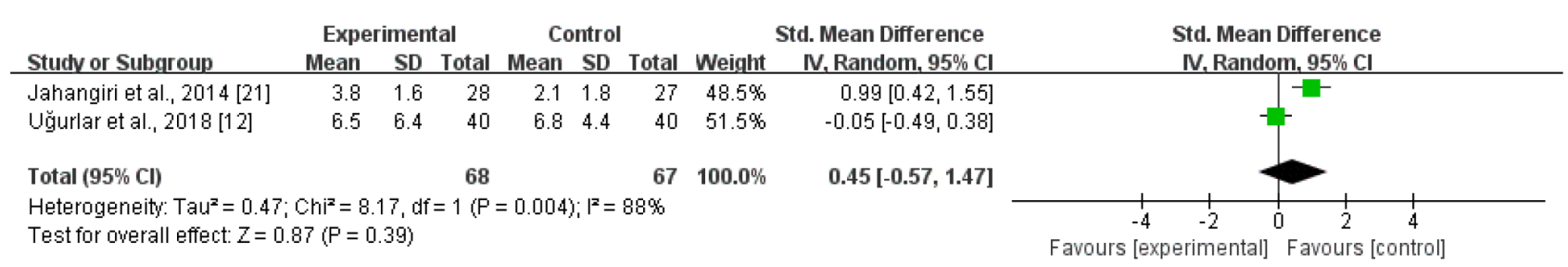

Fig. 4. Forest Plot; (A) saline (B) exercise (C) PRP (D) steroid. Forest plot diagram showing comparisons of VAS for Pain Composite between dextrose prolotherapy and the reference treatments 6 months-1 year. (A) Dextrose vs. Saline on VAS for pain composite 6 months-1 year. (B) Dextrose vs. Exercise on VAS for pain composite 6 months-1 year. (C) Dextrose vs. PRP on VAS for pain composite 6 months-1 year. (D) Dextrose vs. Steroid on VAS for pain composite 6 months-1 year. PRP: platelet-rich plasma, VAS: Visual Analog Scale, Std. Mean difference: standardized mean difference, IV: weighted mean difference, Cl: confidence interval, SD: standard deviation.

had a significant improvement in function compared to the control group $[13,15,17,19-21]$. One study showed functional improvement at 90 days after treatment, but after 360 days, both the prolotherapy and control groups showed similar results [14]. In one study, no significant improvement was noted in any of the groups at the end of the follow-up period [12]. However, unlike other studies which used a dextrose concentration of $10 \%$ or higher, this study only used a $5 \%$ concentration. When used clinically, dextrose concentrations higher than $10 \%$ are partly affected by inflammatory mechanisms, while concentrations less than $10 \%$ are considered noninflammatory $[35,36]$. Considering this, it is possible that a low concentration of dextrose could have affected the therapeutic effect. Although the degree of pain reduction and functional improvement is not completely consistent, there seems to be a correlation 
between the two in the studies that were included in this meta-analysis.

Although there were several positive aspects of our study, there are some limitations. First, despite recent studies being added, the number of trials eligible for inclusion in the meta-analysis was limited. Since the results regarding prolotherapy corresponding to the effects of corticosteroids and PRP were derived by analyzing only two studies, additional studies are needed. Second, there is heterogeneity in the pooled analyses; this is likely attributable to multiple factors, including differences in patient characteristics, control treatment, study design, injection protocol methods, dextrose concentrations, follow-up duration, and outcome assessment methods. A limited number of studies and heterogeneity have inhibited more detailed meta-analyses of subgroups. Third, due to a lack of a uniform longer-term follow-up duration across the studies, pooling of results could only be done with data collected between 6 months and one year of follow-up. Considering that prolotherapy is hypothesized to work by healing and regeneration over several months, reported results of effects may underestimate long-term benefits. Therefore, further studies (including cohort studies) are needed to evaluate the long-term effects. Fourth, since prolotherapy has been shown to have comparable effects to steroid injection and PRP, further studies should be conducted regarding cost effectiveness. Jahangiri et al. [21] compared prolotherapy and corticosteroids and mentioned that there was no significant difference in cost. In previous study, prolotherapy was more effective [14], and has a better cost advantage compared to PRP [37].

In the future, subgroup analysis should be performed to identify patients who respond most favorably to prolotherapy. There are several ways in which treatment strategies can vary; for example, dextrose concentrations/volumes may differ, the interval and total duration of treatment may differ, and the site of injection (intra- or extra-articular areas) may differ. Since there are no clear criteria or standard treatment, this should be discussed in the future. Reducing pain, improving functionality, and increasing patient satisfaction provide a solid foundation for further research in attempt of treatment standardization.

In conclusion, dextrose-based prolotherapy has been shown to have a positive and significantly beneficial effect for patients with chronic musculoskeletal pain, ranging from 6 months to 1 year. There is evidence that dextrose-based prolotherapy has a better therapeutic effect than exercise, and that it has a similar effect compared to PRP and steroid injection. Adequately powered, longer-term trials with uniform endpoints are needed to better elucidate the efficacy of prolotherapy.

\section{ACKNOWLEDGEMENTS}

Special thanks to Jiyeon Ju, and Joonho Cho in contribution to writing this article.

This work was supported by grant from Inje University, 2019 .

\section{CONFLICTS OF INTEREST}

No potential conflict of interest relevant to this article was reported.

\section{AUTHOR CONTRIBUTIONS}

Conceptualization: Geonhyeong Bae, Yunhee Lim. Data curation: Geonhyeong Bae, Sangseok Lee, Woo Yong Lee, Yunhee Lim. Formal analysis: Geonhyeong Bae, Yunhee Lim. Funding acquisition: Yunhee Lim. Writing - original draft: Geonhyeong Bae, Yunhee Lim. Writing - review \& editing: Suyeon Kim, Sangseok Lee, Woo Yong Lee, Yunhee Lim. Supervision: Yunhee Lim.

\section{ORCID}

Geonhyeong Bae, https://orcid.org/0000-0002-8527-0004

Suyeon Kim, https://orcid.org/0000-0002-6167-4952

Sangseok Lee, https://orcid.org/0000-0001-7023-3668

Woo Yong Lee, https://orcid.org/0000-0002-1632-1314

Yunhee Lim, https://orcid.org/0000-0003-2399-4768

\section{REFERENCES}

1. Institute of Medicine of the National Academies. Relieving pain in America: a blueprint for transforming prevention, care, education, and research. Washington, D.C., National Academies Press. 2011. p. 382.

2. Hung CY, Hsiao MY, Chang KV, Han DS, Wang TG. Comparative effectiveness of dextrose prolotherapy versus control injections and exercise in the management of osteoarthritis pain: a systematic review and meta-analysis. J Pain Res 2016; 9: 84757.

3. Ayhan E, Kesmezacar H, Akgun I. Intraarticular injections (cor- 
ticosteroid, hyaluronic acid, platelet rich plasma) for the knee osteoarthritis. World J Orthop 2014; 5: 351-61.

4. Bellamy N, Campbell J, Robinson V, Gee T, Bourne R, Wells G. Intraarticular corticosteroid for treatment of osteoarthritis of the knee. Cochrane Database Syst Rev 2006; (2): CD005328.

5. Aufiero D, Vincent H, Sampson S, Bodor M. Regenerative injection treatment in the spine: review and case series with platelet rich plasma. J Stem Cells Res Rev Rep 2015; 2: 1019.

6. Linetsky FS, Manchikanti L. Regenerative injection therapy for axial pain. Tech Reg Anesth Pain Manag 2005; 9: 40-9.

7. Adams E. Bibliography: prolotherapy for musculoskeletal pain. Boston, Veterans. 2008.

8. Goswami A. Prolotherapy. J Pain Palliat Care Pharmacother 2012; 26: 376-8.

9. Nair LS. Prolotherapy for tissue repair. Transl Res 2011; 158: 129-31.

10. Rabago D, Best TM, Beamsley M, Patterson J. A systematic review of prolotherapy for chronic musculoskeletal pain. Clin J Sport Med 2005; 15: 376-80.

11. Higgins JP, Altman DG, Gøtzsche PC, Jüni P, Moher D, Oxman AD, et al.; Cochrane Bias Methods Group; Cochrane Statistical Methods Group. The Cochrane Collaboration's tool for assessing risk of bias in randomised trials. BMJ 2011; 343: d5928.

12. Uğurlar M, Sönmez MM, Uğurlar ÖY, Adıyeke L, Yıldırım H, Eren OT. Effectiveness of four different treatment modalities in the treatment of chronic plantar fasciitis during a 36-month follow-up period: a randomized controlled trial. J Foot Ankle Surg 2018; 57: 913-8.

13. Seven MM, Ersen O, Akpancar S, Ozkan H, Turkkan S, Yıldız Y, et al. Effectiveness of prolotherapy in the treatment of chronic rotator cuff lesions. Orthop Traumatol Surg Res 2017; 103: 42733.

14. Ersen Ö, Koca K, Akpancar S, Seven MM, Akyıldız F, Yıldız Y, et al. A randomized-controlled trial of prolotherapy injections in the treatment of plantar fasciitis. Turk J Phys Med Rehabil 2017; 64: 59-65.

15. Rabago D, Patterson JJ, Mundt M, Kijowski R, Grettie J, Segal NA, et al. Dextrose prolotherapy for knee osteoarthritis: a randomized controlled trial. Ann Fam Med 2013; 11: 229-37.

16. Bertrand H, Reeves KD, Bennett CJ, Bicknell S, Cheng AL. Dextrose prolotherapy versus control injections in painful rotator cuff tendinopathy. Arch Phys Med Rehabil 2016; 97: 17-25.

17. Kim E, Lee JH. Autologous platelet-rich plasma versus dextrose prolotherapy for the treatment of chronic recalcitrant plantar fasciitis. PM R 2014; 6: 152-8.

18. Yelland MJ, Glasziou PP, Bogduk N, Schluter PJ, McKernon M. Prolotherapy injections, saline injections, and exercises for chronic low-back pain: a randomized trial. Spine (Phila Pa 1976) 2004; 29: 9-16.

19. Reeves KD, Hassanein K. Randomized, prospective, placebo-controlled double-blind study of dextrose prolotherapy for osteoarthritic thumb and finger (DIP, PIP, and trapeziometacarpal) joints: evidence of clinical efficacy. J Altern Complement Med 2000; 6: 311-20.

20. Reeves KD, Hassanein K. Randomized prospective double-blind placebo-controlled study of dextrose prolotherapy for knee osteoarthritis with or without ACL laxity. Altern Ther Health Med 2000 6: 68-74, 77-80.

21. Jahangiri A, Moghaddam FR, Najafi S. Hypertonic dextrose versus corticosteroid local injection for the treatment of osteoarthritis in the first carpometacarpal joint: a double-blind randomized clinical trial. J Orthop Sci 2014; 19: 737-43.

22. Sit RW, Chung VC, Reeves KD, Rabago D, Chan KK, Chan DC, et al. Hypertonic dextrose injections (prolotherapy) in the treatment of symptomatic knee osteoarthritis: a systematic review and meta-analysis. Sci Rep 2016; 6: 25247.

23. Paavola M, Kannus P, Järvinen TA, Järvinen TL, Józsa L, Järvinen $\mathrm{M}$. Treatment of tendon disorders. Is there a role for corticosteroid injection? Foot Ankle Clin 2002; 7: 501-13.

24. Dean BJ, Lostis E, Oakley T, Rombach I, Morrey ME, Carr AJ. The risks and benefits of glucocorticoid treatment for tendinopathy: a systematic review of the effects of local glucocorticoid on tendon. Semin Arthritis Rheum 2014; 43: 570-6.

25. Tempfer H, Gehwolf R, Lehner C, Wagner A, Mtsariashvili M, Bauer HC, et al. Effects of crystalline glucocorticoid triamcinolone acetonide on cultered human supraspinatus tendon cells. Acta Orthop 2009; 80: 357-62.

26. Stannard JP, Bucknell AL. Rupture of the triceps tendon associated with steroid injections. Am J Sports Med 1993; 21: 482-5.

27. Scarpone M, Rabago DP, Zgierska A, Arbogast G, Snell E. The efficacy of prolotherapy for lateral epicondylosis: a pilot study. Clin J Sport Med 2008; 18: 248-54.

28. Okuda Y, Adrogue HJ, Nakajima T, Mizutani M, Asano M, Tachi $\mathrm{Y}$, et al. Increased production of PDGF by angiotensin and high glucose in human vascular endothelium. Life Sci 1996; 59: 1455-61.

29. Oh JH, Ha H, Yu MR, Lee HB. Sequential effects of high glucose on mesangial cell transforming growth factor-beta 1 and fibronectin synthesis. Kidney Int 1998; 54: 1872-8.

30. Di Paolo S, Gesualdo L, Ranieri E, Grandaliano G, Schena FP. High glucose concentration induces the overexpression of transforming growth factor-beta through the activation of a platelet-derived growth factor loop in human mesangial cells. Am J Pathol 1996; 149: 2095-106. 
31. Kampa RJ, Connell DA. Treatment of tendinopathy: is there a role for autologous whole blood and platelet rich plasma injection? Int J Clin Pract 2010; 64: 1813-23.

32. Sampson S, Gerhardt M, Mandelbaum B. Platelet rich plasma injection grafts for musculoskeletal injuries: a review. Curr Rev Musculoskelet Med 2008; 1: 165-74.

33. Foster TE, Puskas BL, Mandelbaum BR, Gerhardt MB, Rodeo SA. Platelet-rich plasma: from basic science to clinical applications. Am J Sports Med 2009; 37: 2259-72.

34. de Mos M, van der Windt AE, Jahr H, van Schie HT, Weinans H,
Verhaar JA, et al. Can platelet-rich plasma enhance tendon repair? A cell culture study. Am J Sports Med 2008; 36: 1171-8.

35. Jensen KT, Rabago DP, Best TM, Patterson JJ, Vanderby R Jr. Early inflammatory response of knee ligaments to prolotherapy in a rat model. J Orthop Res 2008; 26: 816-23.

36. Jensen KT, Rabago DP, Best TM, Patterson JJ, Vanderby R Jr. Response of knee ligaments to prolotherapy in a rat injury model. Am J Sports Med 2008; 36: 1347-57.

37. Mei-Dan O, Mann G, Maffulli N. Platelet-rich plasma: any substance into it? Br J Sports Med 2010; 44: 618-9. 